\title{
Apoptosis of Circulating Heterophils; Implications for the Interpretation of the Heterophil/Lymphocyte Ratio
}

Paul F. Cotter

Cotter Laboratory, Arlington, MA 02476, USA

Article Info

\section{Article Notes}

Received: September 01, 2021

Accepted: October 07, 2021

\section{*Correspondence:}

*Dr. Paul F. Cotter, Cotter Laboratory, Arlington, MA 02476, USA; Tel: 781646 8976; Email: kamcotter@juno.com.

(c) 2021 Cotter PF. This article is distributed under the terms of the Creative Commons Attribution 4.0 International License.

\section{Keywords}

Apoptosis

Arneth index

Atypical heterophil

Hemogram

$\mathrm{H} / \mathrm{L}$ ratio

Stress

\section{ABSTRACT}

The aim is to demonstrate a variety of apoptotic heterophils (equivalents of mammalian neutrophils) occurring in hemograms of 50 apparently healthy pullets housed in cages. These atypical cells are found in blood with normal total white cell levels (TWBC, $\sim 20 \mathrm{~K}$ ) and in those ranging up to $100(\mathrm{~K})$ a leukocytosis/leukemoid reaction level. Conversely heterophil/lymphocyte $(\mathrm{H} / \mathrm{L})$ ratios in all hens ranged between $0.14-0.50$ (homeostasis). The Arneth index (a heterophil age measure) of 1.8 computed using only intact heterophils indicated a "left-shift", suggesting inflammation even in the context of a normal hemogram.

The results: atypical heterophils with condensed (pyknotic) and fragmented nuclei (karyorrhexis), ruffled cytoplasmic membranes (zeiosis), apoptotic body formation, and other characteristics of apoptosis circulated in the blood of the study hens. Highly unusual cells as a monocyte containing a phagocytosed apoptotic body composed of heterophil granules, and a Mott cell (atypical plasmacyte) were also found.

More importantly, heterophils displaying thin, swollen and externalized nuclei suggestive of an unusual form of karyolysis were present. These cells were often seen in the company of free or RBC associated bacteria and fungi. There were also two examples of bacteria phagocytosed by apoptotic cells.

Other types of apoptotic cells were in 16/50 (32\%) of hens from the study sample. These included small and medium size lymphocytes, monocytes, and metamyelocytes. Frank evidence of bacteremia $(5 / 50,10 \%)$ fungemia $(4 / 50$, $8 \%)$ and both $(1 / 50,2 \%)$ were detected. This suggests a relation between hematological atypia and the presence of circulating microorganisms possibly mediated through the release of toxins. Atypia of the circulation are sentinels, an alert to investigators or clinicians who are interested in the relation between the composition of blood and stress measurement or disease.

Conclusions: these observations apply to apparent morphologic plasticity of the heterophil, its role in the innate immune system, and its use in determining stress. In the cases described here the initial computation of the $\mathrm{H} / \mathrm{L}$ ratios suggested the absence of stress. Moreover, this occurred in the context of either a normal or a high TWBC. However, heterophil age indicated a left-shift; a population predominated by young cells. This situation occurs when there is a demand to replace cells that have left the circulation either due to inflamed tissues or to death while circulating. Therefore, circulating apoptotic cells suggest stress even when the $\mathrm{H} / \mathrm{L}$ is low and the TWBC is normal.

\section{Introduction}

The heterophil, regarded as the avian equivalent of the mammalian neutrophil, is a cell with a marked capacity for phagocytosis. It is an essential component of the innate immune system ${ }^{1}$. Its basic biology and some of its morphological variation was extensively reviewed by Maxwell and Robertson ${ }^{2}$. A more recent description of heterophil 
biology was provided by Genovese ${ }^{3}$ who emphasized receptors, signaling, antimicrobial products, cytokine and chemokine production, and the effects of genetic selection.

\section{Heterophil Death by Apoptosis}

The process of apoptosis (programmed cell death) describes the end stages of the normal life history of cells such as the heterophil. Beginning with production in the bone marrow from myelocyte precursors, they progress there to the mature stage before entering the circulation. Later, after exiting the blood, the heterophil now resides in normal tissue, or drawn by chemotaxis, occupies a site of inflammation. Once the inflammation is resolved effete heterophils are removed by resident macrophages through phagocytosis. Removal is in a way that prevents release of potentially injurious substances paralleling the fate of mammalian neutrophils ${ }^{4}$. The entire life-history, from production in the bone marrow to death in the tissues, is completed in a few days.

When it is their time, heterophils die by a genetically guided mechanism known as apoptosis (programmed cell death). It begins with cell shrinkage, chromatin condensation, nuclear fragmentation (pyknosis) membrane ruffling or blebbing a process described as zeiosis. Heterophil membrane fragments may get packaged into apoptotic bodies; as occurs with mammalian neutrophils ${ }^{5}$.

The differences between apoptosis and necrosis (traumatic death) are now well known. Using electron microscopy, Shini, et al. ${ }^{6}$ showed the differential effects of injected corticosterone or LPS on the death of chicken heterophils by apoptosis and necrosis. However, death by necrosis (necroptosis) has emerged as an alternate form of programmed cell death whose activation might have important biological consequences, including the induction of an inflammatory response ${ }^{7}$.

\section{Morphological Study}

Heterophils illustrated by Lucas and Jamroz ${ }^{8}$ (p. 75, Figures 159 -167) indicate variation of their nuclei, cytoplasmic granules, and size. However, no examples of apoptotic cells are included. Therefore, a description of morphological changes of circulating heterophils suggesting this process would aid those interested in the role of the heterophil in stress and disease. These cells were detected by light microscopic examination of blood films from apparently healthy hens. Collectively they indicate apoptosis albeit occurring at an inappropriate time. Additionally, some cells displayed a death mechanism in which destruction of the nucleus (karyolysis) is an early step. To the knowledge of the author, such events have not been described elsewhere.

\section{Materials and Methods}

\section{Chickens}

Fifty pullets (18 wk) of a Lohmann White Egg commercial type (LSL) housed in commercial cages (CC) at 6 per unit are the source of blood samples. Vaccines against Marek's disease and laryngotracheitis were applied at the hatchery; and additional vaccinations were given later. They were reared using a typical program for the management of commercial hens as described by Jones ${ }^{9}$ et al. The experimental protocol was approved by the Michigan State University Institutional Animal Care and Use Committee.

\section{Blood and Stain Procedures}

Samples, 1 to $3 \mathrm{~mL}$, collected from wing veins into EDTA tubes, were obtained at $18 \mathrm{wk}$ (placement). Monolayer films made by spreading approximately $3 \mu \mathrm{L}$ of blood across alcohol cleaned glass microscope slides were dried immediately by a hot air stream. Slides were then immersed in $95 \%$ ethanol and postfixed for 10 to $15 \mathrm{~min}$. Films were stained by using an in-house version of Wright's method followed by a brief secondary exposure to Giemsa (Sigma) after Hewitt ${ }^{10}$ and Smith ${ }^{11}$. Films were made within $24 \mathrm{~h}$ of collection to minimize the effects of storage.

Gram stains (Fisher, Protocol ${ }^{\mathrm{R}}$ Reagents) were applied to several slides after extraction of the Wright-Giemsa by repeated soaks with $95 \% \mathrm{EtOH}$.

\section{Light Microscopy and Photomicrographs}

An Olympus CX-41(Olympus America, Center Valley, PA) fitted with Plan N 40×, 0.65 Numerical Aperture (N.A.) dry, and Plan N, 1.25 N.A. 100× (oil) objectives. Images were captured by an Infinity-2 1.4 Megapixel CCD USB 2.0 Camera and processed with Infinity Analyze software (Release 5.0.3, Lumenera Inc., Ottawa, Ontario, Canada). Magnification was 40x for standard differential counts (SDC) and selected images were photograph at 100x (oil).

\section{Differential Counts and TWBC}

Following the recommendation of Lucas and Jamroz a standard differential count (SDC) is composed of two parallel sweeps totaling at least 400 leukocytes. The first starts near Vernier gauge position $5 \mathrm{~mm}$ of the microscope's mechanical stage and continues in one direction until at least 200 leukocytes are counted. A second count starts near the $10 \mathrm{~mm}$ position and proceeds as above. Leukocytes are sorted on morphological criteria described in $^{8}$ and Cotter ${ }^{12-14}$ into the following categories: small "resting" lymphocytes (Ls) medium/large and reactive lymphocytes (Lm), monocytes (Mn), heterophils (HC, "classic" having red rod-shaped cytoplasmic granules, "typical" (HT) having weakly stained granules providing a reticular cytoplasmic 
appearance, or rarely as "variant" types (HV) having orange spherical granules) metamyelocytes ( $\mathrm{mtm})$, basophils $(\mathrm{Ba})$, and eosinophils (Eo).

TWBC were estimated from the same slides and fields as SDC's by a method described in Campbell and Ellis ${ }^{15}$.

\section{Arneth Index}

The Arneth index has long been used to indicate age of cells whose nuclei are lobed (A. G. N. ${ }^{16}$ describes its history). Older cells have more nuclear lobes than young cells and from a population status the lobe average can be used to indicate a "left" or "right" shift. The index was computed by sorting (at 100x, oil) standard sized HC (D $9 \mu \mathrm{m}$ ) from the original SDC into groups based on the lobe number using the computation method of Lucas and Jamroz ${ }^{8}$ (p. 85). Apoptotic HC, or dwarf cells ( $D \sim 7 \mu \mathrm{m}$ ) were excluded. Afterward, atypical heterophils were added to the HC group either as apoptotic bodies (cytoplasmic granules without a nucleus; 0-lobes) or with the "Apo" group if a nucleus or its remnant was present. Figure 1 describes the Arneth count (standard HC having: 1, Band (assigned 1.5) 2, or 3 lobed nuclei) including atypical cells.

\section{Results}

\section{Morphology of Classic \& Variant Heterophils}

A representative field containing intact cells; a solitary HC, thrombocytes (Th) and a solitary HV is shown in Figure 2. HV nuclei are typically rounder than the elliptical or irregular shapes of HC nuclei. HV chromatin generally appears more condensed than HC chromatin because more clear spaces are seen within nuclear lobes, but these differences are not absolute. HV granules are orange and distinctly circular in contrast to the red ellipsoids of HC.

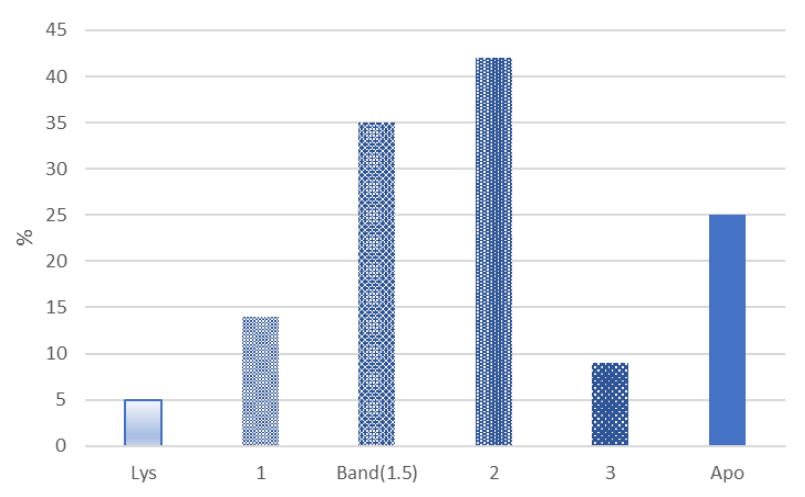

Figure 1. Counts (\%) of the nuclei of 100 normal Classic Heterophils (HC) were sorted into groups based nuclear lobe number; 1 , Band (1.5) 2, or 3. Atypical HC's (apoptotic bodies) with lysed nuclei were grouped in the 0 -lobe (Lys) category; others displaying condensed chromatin or pyknosis, and blebbing were grouped in the apoptosis (Apo) category and later added. The Arneth index (1.8) did not include either the 0 -lobe or Apo groups. Atypical HC's (Lys + Apo) were $30 / 130$ (23.3\%).

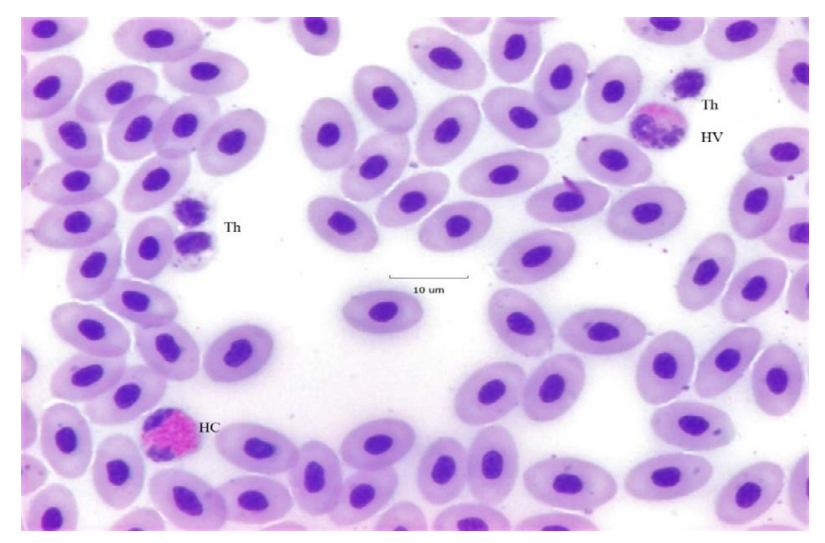

Figure 2. A 40x field displaying a standard size ( $9 \mu \mathrm{m}$ diameter) classic heterophil $(\mathrm{HC})$ at the lower left. The nucleus has two fully stained lobes one of which (top) is elliptical, the other (bottom) has an irregular shape. The red cytoplasmic granules are elongated and display deep red circular central bodies. A variant heterophil (HV) seen at the top right with two nearly circular nuclear lobes is easily differentiated from an HC. HV chromatin is more compact than of $\mathrm{HC}$ as is indicated by larger empty spaces within the nucleus. The cytoplasmic granules of the HV are circular in contrast to the ellipses of the HC. In many instances HV granules display an orange hue and are restricted to one side of the cell as is the case here. Cells labelled Th are (reactive) thrombocytes known to be related to the blood clotting mechanism. The background erythrocytes are $\sim 10 \mu \mathrm{m}$ in length and several located at the lower right appear to display surface associated bacteria.

Moreover, HV granules are typically distributed to a side of the cell opposite the nucleus. HV resemble an example included with eosinophils, whose granules are red, in Lucas and Jamroz ${ }^{8}$ (p. 77, cell \#180). However, in this study HV comprised $\sim 9.4 \%$ (208/2215) of all granulocytes; a frequency more than twice values given by Lucas and Jamroz $^{8}(1.4-3.9 \%)$ for chicken eosinophils (p. 216, Table 17). Neither the HC nor the HV of show signs of apoptosis. Therefore, the intact HC and HV of Figure 2 act as reference cells against which atypical cells can be compared.

The background erythrocytes whose long axis averages $10.7(+/-0.7 \mu \mathrm{m}, \mathrm{N}=10)$ are unremarkable. Therefore, RBC's may be used as a convenient micrometer if one is not included with photomicrographs. The cytoplasm of the elliptical Th appears to contain multiple specific granules and vacuoles, and so would be grouped with "reactive types" by the criteria of Lucas and Jamroz (p. 38).

\section{Age of Heterophils}

The number of lobes of heterophil nuclei is taken as an estimate of their age. Single lobed cells are the youngest and those with two, three, or more are regarded as older cells. A "band cell" a young heterophil with one elongated nuclear lobe, or one in which the two nuclear lobes are in close proximity, is assigned 1.5 lobes (Figure 3 ). The distribution computed by the average product of the lobe number $\mathrm{x}$ frequency (Arneth index) estimates population 


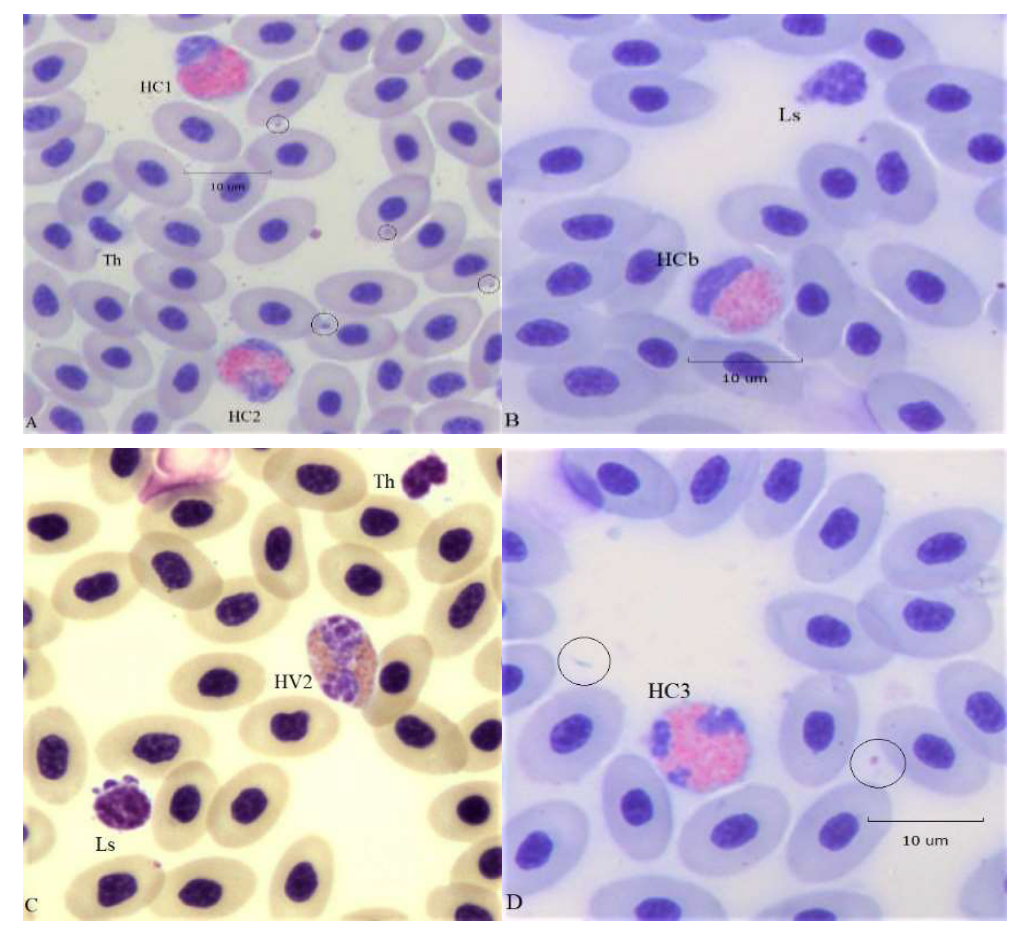

Figure 3. Four 100x (oil) fields displaying standard size classic (HC) and variant (HV) heterophils of various "ages": panel A, HC1, HC2; 1and 2 lobed HC types, panel B) 1.5 lobe (HC band), panel C) 2 lobed HV type; and panel D) 3 lobe HC type. Ls are small lymphocytes and Th are thrombocytes. Arrows and circles locate free or cell associated bacteria commonly seen in the hemograms of this study.

age. Indices of $<2.3$ describe a "left-shift", those at $\sim 2.5$ are average, and $>2.7$ indicates a shift to the right (p. 85, Lucas and Jamroz ${ }^{8}$. Figure 1 gives the frequency distribution of the Arneth index computed from measuring 100 intact HC not showing alterations of the nuclei suggesting apoptosis. The figure also gives the frequency of a zero-lobe group (granules without nuclei or very faint remnants, see Figure 6) and an 'Apo' group containing apoptotic cells, also in Figure 6. The index (1.8) indicates a "left-shift" population assumed to result from a demand to replace effete cells and those removed from the circulation due to inflammation. It is not known if an HC can proceed directly from the 1 lobe stage to lysis or apoptosis or must first advance to a higher lobe number.

\section{Apoptosis of Heterophils}

Apoptosis is programmed cell death, a regulated process during which cells shrink, chromatin condenses, the nucleus becomes fragmented, and the cytoplasmic membrane develops out pocketing (blebs). Shidham and Swami ${ }^{16}$ described apoptotic changes of circulating human neutrophils and other leukocytes associated with infection. Heterophils displaying similar apoptotic changes are illustrated in Figure 4.

\section{Karyolysis of HC}

Changes of nuclei of recognized by light microscopy are condensation (pyknosis) fragmentation (karyorrhexis) and dissolution (karyolysis). The nuclei and mitochondria of cells examined by electron microscopy undergoing necroptosis become swollen and the cell membrane becomes permeable ${ }^{17}$. Necrosis is thought of as a random process, but apoptosis and necroptosis are distinct guided programs leading to cellular death. In inflamed tissues effete $\mathrm{HC}$ are believed to die by apoptosis, and are removed by resident macrophages.

HC with swollen externalized nuclei were in the circulation in the company of intact and apoptotic HC (Figure 5). In some instances, the nuclei of these cells remained associated with the cytoplasm (panels A and B). In other cases, nuclei became so thin as to be barely visible (panels C and D). Moreover, some cytoplasmic granules appeared to have escaped, suggesting increased permeability of the cell membrane (panels A and C). These changes are not likely the result of technical artifacts occurring during preparation of the slide because there was no evidence of smudging of nearby RBC. Therefore, it is suggested that these represent a form of $\mathrm{HC}$ necroptosis.

\section{HC Phagocytosis}

Further examples of necroptosis appear in Figure 6. In panel A, the HC nucleus has disappeared completely leaving only cytoplasmic granules apparently still held in place by the remnants of the cell membrane. A few HC cytoplasmic granules have been phagocytosed by a monocyte, panel B. An apoptotic HC (top) containing a phagocytosed bacterium (arrow) appears in field with an intact HC 


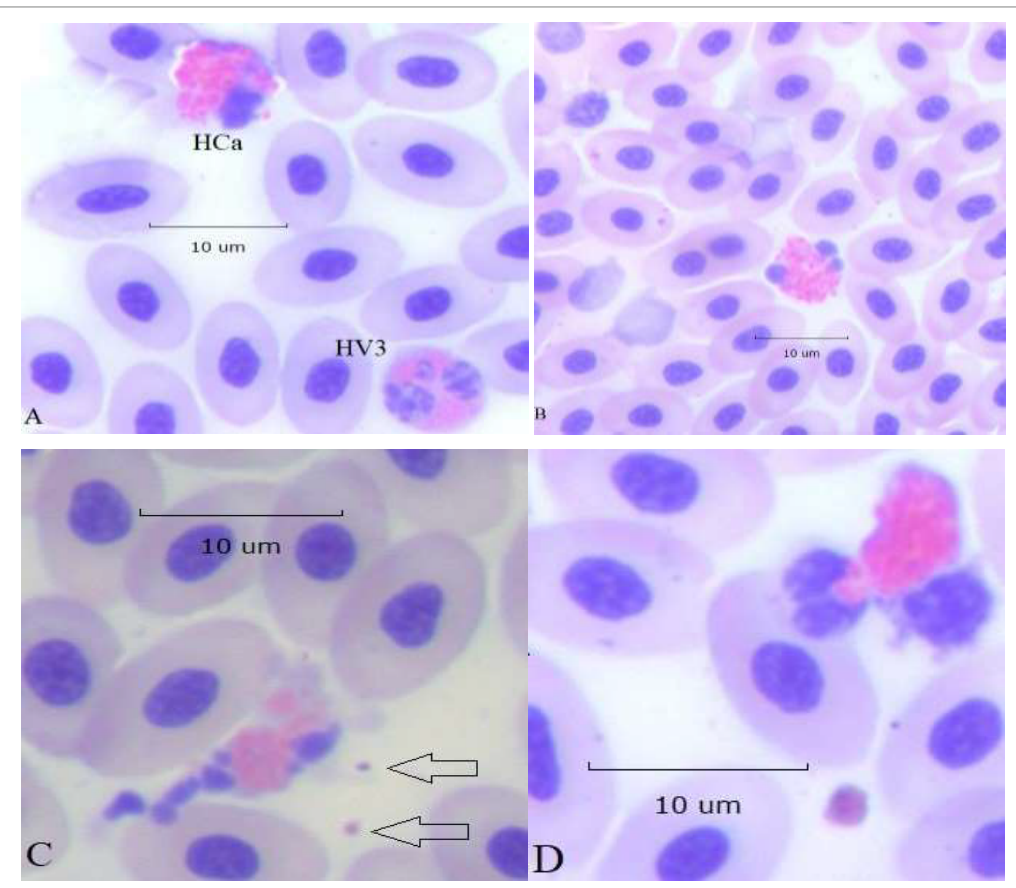

Figure 4. Apoptotic changes of HC heterophils (100x): panel A, HC with early nuclear condensation (pyknosis) and cytoplasmic blebbing, top left, normal 3 lobe HV, bottom right. Panel B, HC with 3 condensed nuclear lobes. Panel C, HC with fully fragmented nuclear lobes and severe membrane blebbing. Two encapsulated bacteria are at the right of the HC (arrows). Panel D, HC in which two nuclear lobes are within a single membrane bleb (zeiosis) also containing a few cytoplasmic granules.
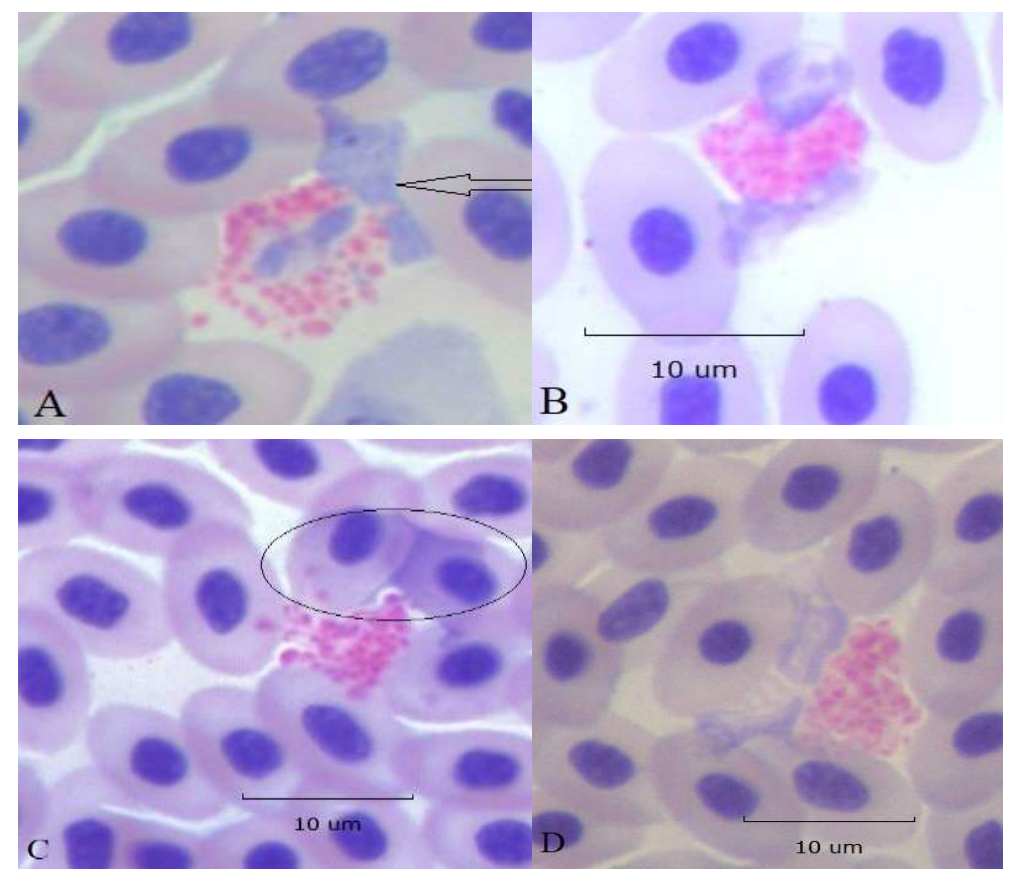

Figure 5. Karyolysis of HC (100x). Early stages when fading swollen nuclei remain in association with cytoplasm, panels A and B. In panel A one nuclear lobe is already extracellular (arrow). Later stages when nuclei have separated from cytoplasm and are seen as transparent veils (encircled) over adjacent RBC's, panel C. Panel D, extracellular nuclear remnants are situated between the cytoplasmic granules and an adjacent RBC. All lytic events are localized to HC nuclei and do not involve nearby cells; ruling out technical artifacts.

(bottom right) panel C. In panel D an apoptotic HC with condensed nuclear lobes and membrane zeiosis contains 3 phagocytosed bacteria (large arrow) free bacteria are seen nearby (small arrows).

\section{Apoptosis of Other Leukocytes}

Apoptotic leukocytes found in the hemogram of another hen of the same study appear in Figure 7. Four Ls displaying various degrees of nuclear condensation and zeiosis occupy 


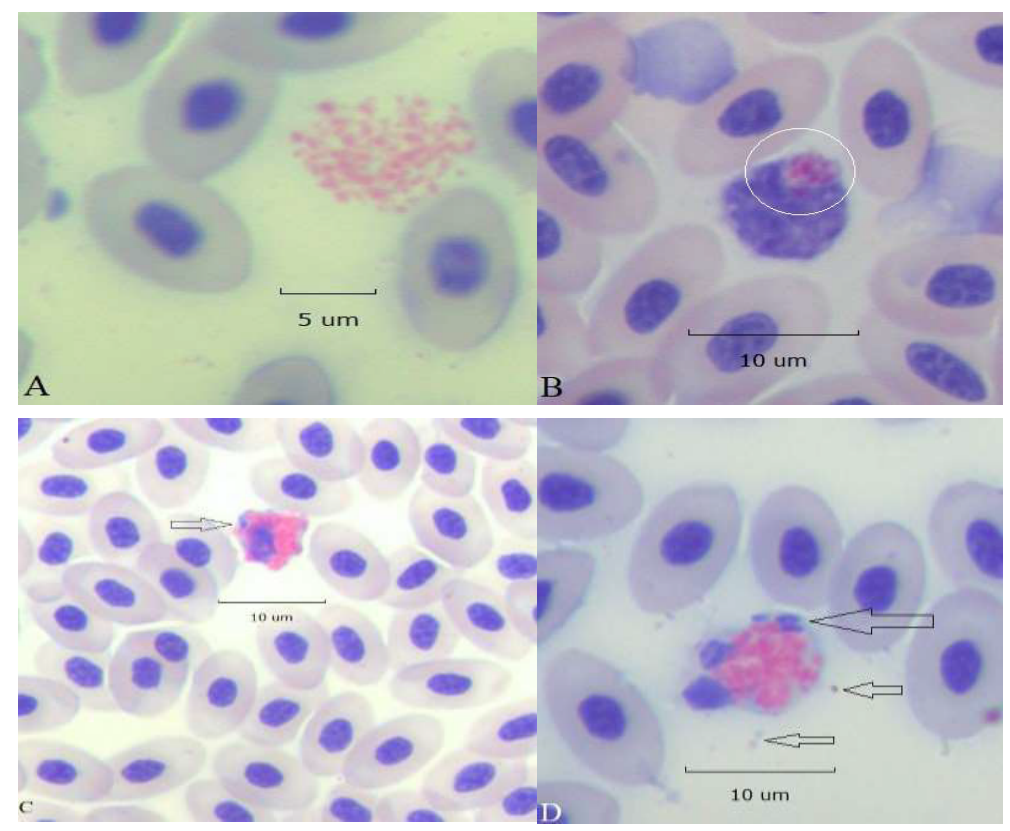

Figure 6. A cluster of HC cytoplasmic granules free of nuclear remnants, panel A. A monocyte with a phagocytosed apoptotic body composed of HC cytoplasmic granules (circled), panel B. An apoptotic HC displays a ruffled edge, a partially condensed nucleus, and a phagocytosed bacillus (arrow) panel C. Panel D, an HC with two pyknotic nuclear lobes contains 3 phagocytosed bacilli (large arrow) free bacteria are nearby (small arrows; all 100x).

Table 1. Average ( $2 \times 200$ cell) standard differential count (SDC) in \% of total white blood cells (TWBC(K) thousand) and heterophil/lymphocyte ratios for blood films providing figures and the sample flock ave.

\begin{tabular}{|c|c|c|c|c|c|c|c|c|c|c|c|c|c|c|}
\hline Fig & $H^{\mathrm{a}}$ & HV & $\mathrm{HC}$ & Ls & $\mathrm{Lm}$ & NK & $\mathrm{mtm}$ & $M n$ & $\mathrm{Ba}$ & Eo & H/L 1 & H/L 2 & $\Delta H / L$ & TWBC(K) \\
\hline $1-6$ & 23.9 & 1.9 & 0.0 & 51.9 & 13.0 & 0.0 & 2.4 & 2.2 & 4.6 & 0.0 & 0.50 & 0.40 & 0.1 & 21.3 \\
\hline 7 & 18.5 & 0.0 & 0.0 & 68.4 & 9.7 & 1.2 & 0.2 & 0.0 & 1.7 & 0.2 & 0.31 & 0.25 & 0.06 & 100 \\
\hline 8 & 4.7 & 0.5 & 4.8 & 65.4 & 6.5 & 0.3 & 0.3 & 1.3 & 2.9 & 0.2 & 0.07 & 0.06 & 0.01 & 20.7 \\
\hline Flock Ave. & 5.0 & 0.8 & 4.9 & 75.9 & 5.5 & 0.4 & 0.4 & 2.4 & 3.0 & 0.1 & 0.15 & 0.14 & 0.01 & 56.1 \\
\hline
\end{tabular}

${ }^{a}$ Abbreviations: Cells: $\mathrm{H}$, heterophil (HT typical, HV variant, $\mathrm{HC}$ classic) Ls, small lymphocyte $6 \mu \mathrm{M}$ diameter, Lm, medium and large lymphocyte (diameter $8-10 \mu \mathrm{M}$ ), NK natural killer, mtm metamyelocyte, Mn monocyte, Ba basophil, Eo eosinophil. H/L $1=(\mathrm{HT}+\mathrm{HV}+\mathrm{HC}) / \mathrm{Ls} ; \mathrm{H} / \mathrm{L} 1=$ $(H T+H V+H C) /(L s+L m), \Delta H / L=H / L 1-H / L 2 ; T W B C$ total white blood cells per cubic $\mu \mathrm{L}$ in thousands (K).

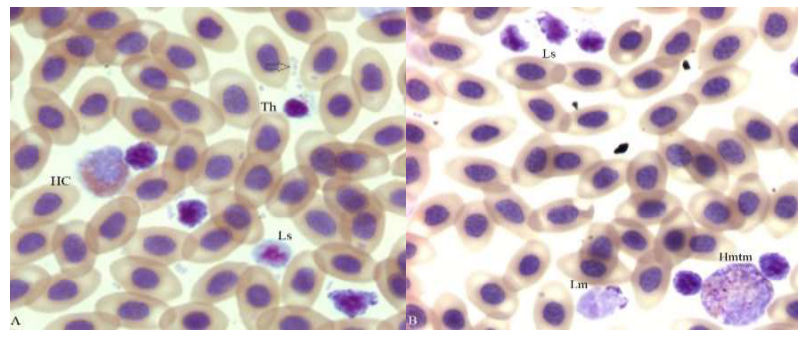

Figure 7. Four apoptotic small lymphocytes (LS) with condensed nuclei and various degrees of membrane blebs (zeiosis), a reactive thrombocyte (Th) an HC, and bacteria (arrow) are in panel A. A group of 3 apoptotic $L s$ is at the top, a medium size lymphocyte (Lm, bottom center) a metamyelocyte $(\mathrm{Hmtm})$ rosette with 2 attached apoptotic Ls (bottom right) are in panel B (100x).

the diagonal of panel A. One Ls displaying minimal signs of nuclear condensation and zeiosis is attached to an intact HC. Condensation and zeiosis are more apparent in the other 3 Ls. Free and RBC associated bacilli are distributed throughout the field (arrow and elsewhere). Additional examples of apoptotic Ls exhibiting both condensed nuclei and zeiosis appear in panel B at the top; an apoptotic Lm with conspicuous zeiosis is in the middle bottom. The 2 Ls attached to the Mn at the bottom right have minimally condensed nuclei and zeiosis. The metamyelocyte ( $\mathrm{mtm}$ ) cytoplasm contains a few deep-purple "toxic granules" and vacuoles (clear spaces). The TWBC of this hen, at $100 \mathrm{~K}$, is in the leukemoid reaction range unlike the hen used for Figures 1-5 whose TWBC at $21.3 \mathrm{~K}$ was in the normal range. However, the H/L of both hens appears to indicate nonstress (Table 1).

\section{Microbiology}

Examples of bacteria seen in the blood films of the study hens are given in Figure 8. Included are several forms of bacteria and fungal elements (conidia). Multiple bacterial forms (polymicrobial) were detected in all bacteremic hens (Panels A, B and C) and several fungal elements were seen. One example of fungal conidia in given in Panel D. 


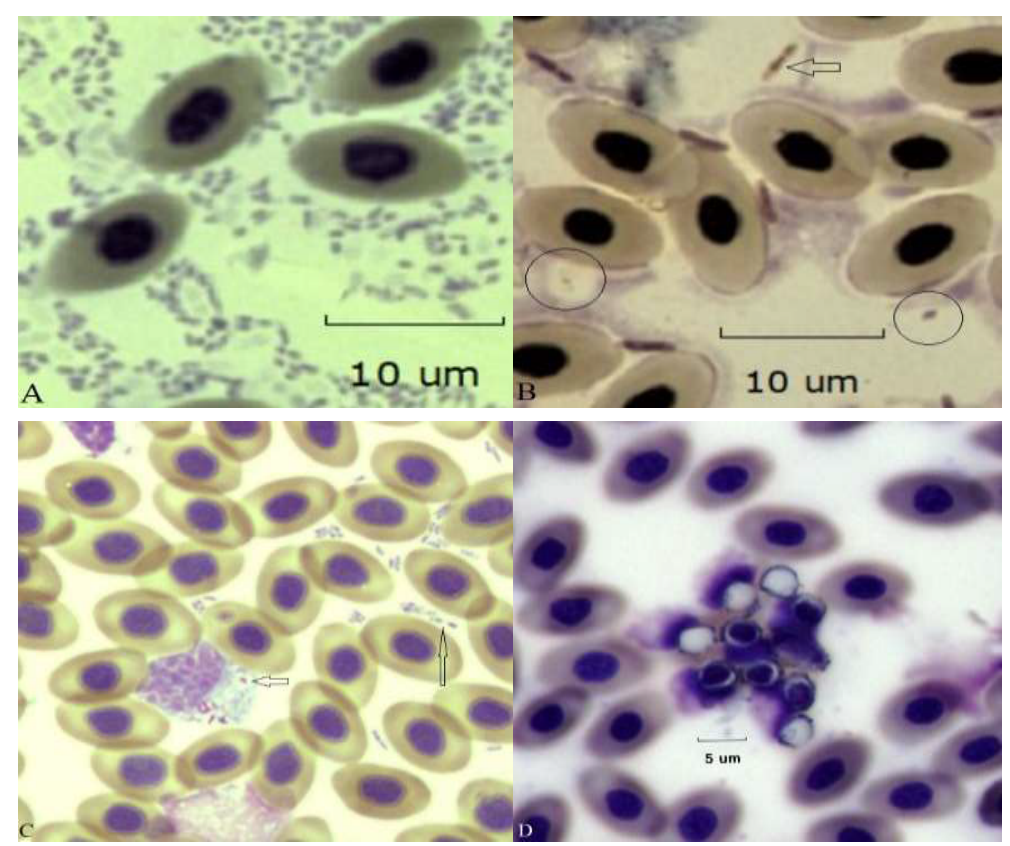

Figure 8. Examples of bacteremia/fungemia in four different hens of the study. Panel A. Polymicrobial bacteremia comprised of small ( 1 $\mu \mathrm{m}$ ) bacilli. Panel B. Large bacilli ( $4 \mu \mathrm{m}$, arrow) and smaller encapsulated bacilli are circled (Gram-stain). Panel C. Small ( $1 \mu \mathrm{m})$ bacilli are both free swimming (possibly containing an endospore, large arrow) and phagocytosed by a monocytic cell (small arrow). Panel D. A cluster of conidia seen on a slightly out-of-focus RBC background photographed to preserve fungal detail.

\section{Discussion and Conclusions}

The present observations indicate heterogeneity among circulating heterophils involving both cell age as measured by nuclear segmentation, and their condition. Heterophils displaying morphologic features consistent with apoptosis were found among intact types having 1, 2 and 3 nuclear lobes. The atypical types had ruffled membranes (zeiosis), condensed (pyknosis) and fragmented nuclei (karyorrhexis). In addition, the nuclei of some heterophils showed signs of expulsion from the cytoplasm and lysis. Since specific biological markers capable of differentiating apoptosis/necrosis were beyond the scope of this study, the molecular mechanisms producing these atypical forms remain to be determined.

Lucas $^{8}$ shows a number of technical artifacts "smudge cells" with disrupted membranes and lysed nuclei in his figures along with intact cells. The atypical cells illustrated, here, in Figures 2 - 6 are not technical artifacts as they are surrounded by erythrocytes not showing signs of disruption or lysis. Therefore, they represent an extension of differences among (HC) heterophils already separated from HT, and HV types ${ }^{18-20}$. Figure 7 displays Ls and Lm with apoptotic features.

It is presumed that heterophils behave similarly to mammalian neutrophils by living only briefly in the circulation before extravasation and ultimate death in the tissues. However, there are no studies describing heterophil longevity (Personal communication, K. Genovese, U.S.D.A).
As is the case with neutrophils ${ }^{21}$ apoptotic heterophils in the circulation imply stress and infer disease. Here, they are seen in the presence of bacteria and fungi, likely sources of toxins.

Three aspects of the present study relate directly to the widely used heterophil/lymphocyte method for stress determination ${ }^{22}$ (reviewed in Davis ${ }^{23}$ ) First, nuclear segmentation is a measure of cell age. At $<2$ the Arneth index (1.8, Figure 1) indicates a young population of cells in the circulation; brought there by physiologic demand. The author is unaware of any $\mathrm{H} / \mathrm{L}$ study that also gives the Arneth index of the cells used for its computation. Therefore, a low or non-stress H/L computed from a "left-shift" population cannot represent the same physiologic situation as one computed from "average-age" or "right-shift" types. Moreover, when apoptotic lymphocytes (Ls and Lm) are also present, as was the case here (Figure 7) a further difficulty arises. Moreover, it is unlikely that apoptotic cells would be recognized in $\mathrm{H} / \mathrm{L}$ determinations by hemacytometer counts or even by automated procedures.

The heterophil is clearly a cell with an important role in innate immunity, historically known for its phagocytic prowess. Therefore, it is not surprising to find phagocytic heterophils in the circulation when there is a bacteremia and fungemia. However, what is truly surprising is to see phagocytic cells that also display signs of apoptosis.

\section{Author contributions}

$\mathrm{PFC}$ is the sole author 


\section{Conflicts of Interest}

The author declares that the research was conducted in the absence of any commercial or financial relationships that could be construed as a potential conflict of interest.

\section{Ethical Approval}

The experimental protocol was approved by the Michigan State University Institutional Animal Care and Use Committee.

\section{Consent to Participate}

Not applicable.

\section{Consent to Publication}

Not applicable.

\section{Availability of Data and Materials}

All are included in the manuscript

\section{Funding}

None

\section{References}

1. Harmon BG, 1998. Avian heterophils in inflammation and disease resistance. Poult. Sci. 77: 972-977. PMID: 9657606 DOI: 10.1093/ ps/77.7.972

2. Maxwell M, and G. Robertson,1998. The avian heterophil leucocyte: A review. World's Poultry Science Journal, 54(2), 155-178. doi: 10.1079/WPS19980012.

3. Genovese KJ, He H, Swaggerty CL, et al, 2013. The avian heterophil. Dev. Comp. Immunol. 41: 334-40. doi: 10.1016/j.dci.2013.03.021. PMID: 23583524.

4. Rungelrath V, Kobayashi SD, DeLeo FR. 2020. Neutrophils in innate immunity and systems biology-level approaches. Wiley Interdiscip. Rev. Syst. Biol. Med. 2020 Jan; 12(1): e1458. doi: 10.1002/wsbm.1458. PMID: 31218817; PMCID: PMC6898734.

5. Squier MK, Sehnert AJ, Cohen JJ. 1995. Apoptosis in leukocytes. J. Leukoc. Biol. 57(1): 2-10. doi: 10.1002/jlb.57.1.2. PMID: 7829971.

6. Shini S, Kaiser P, Shini A, et al. 2008. Differential alterations in ultrastructural morphology of chicken heterophils and lymphocytes induced by corticosterone and lipopolysaccharide. Vet. Immunol. and Immunopath. 122: 83-93. PMID: 18045696 DOI: 10.1016/j. vetimm.2007.10.009
7. Edinger AL, Thompson CB, 2004. Death by design: apoptosis, necrosis and autophagy, Current Opinion in Cell Biology, 16(6): 663-669, ISSN 0955-0674, https://doi.org/10.1016/j.ceb.2004.09.011.

8. Lucas AM, and Jamroz C. 1961. Atlas of Avian Hematology, Monograph 25. USDA, Washington, DC.

9. Jones DR, Karcher DM, and Abdo Z, 2014. Effect of a commercial housing system on egg quality during extended storage. Poult. Sci. 93: 1282-1288. 10.3382/ps. 2013-03631.

10. Hewitt R, 1942. Studies on the host-parasite relationship in untreated infections with Plasmodium lophurae in ducks. Am. J. Hyg. 36: 6-42.

11. Smith EA, 1947. Certain characteristics of the leukocytes of guinea pig blood with particular reference to the Kurloff body. Blood 2: 125-147.

12. Cotter PF, 2015. An examination of the utility of heterophil-lymphocyte ratios in assessing stress of caged hens. Poult. Sci. 94:512-517 http:// dx.doi.org/10.3382/ps/peu009.

13. Cotter PF. 2015. Are peripheral Mott cells an indication of stress or inefficient immunity? Poult. Sci. 94: 1433-1438 http://dx.doi. org/10.3382/ps/pev053.

14. Cotter PF. 2015, Atypical lymphocytes and leukocytes in the peripheral circulation of caged hens. Poult. Sci. 94:1439-1445 http:// dx.doi.org/10.3382/ps/pev157.

15. Campbell TW and Ellis CK, 2007. Avian and exotic animal hematology and cytology 3rd ed. Blackwell Pub. Ames, Iowa 50014, USA.

16. Shidham VB, Swami VK, 2000. Evaluation of apoptotic leukocytes in peripheral blood smears. Arch. Pathol. Lab. Med. 124: 1291-1294. doi: 10.1043/0003-9985(2000)124<1291: EOALIP>2.0.CO;2.

17. Liu L, Fan J, Ai G, et al. 2019. Berberine in combination with cisplatin induces necroptosis and apoptosis in ovarian cancer cells. Biol. Res. 52:37 https://doi.org/10.1186/s40659-019-0243-6

18. Cotter PF and Bakst MR. 2017. A comparison of Mott cell morphology of three avian species. II. - Bad behavior by plasmacytes? Poult. Sci. 96: 325-331 http://dx.doi.org/10.3382/ps/pew288.

19. Cotter PF and Heller ED. 2016. Complex hemograms of isolator raised specific pathogen free (SPF) chicks. Int. Jour. of Poult. Sci., 15: 211217.DOI: 10.3923 /ijps.2016.211.217.

20. Cotter PF. 2021. Atypical hemograms of the commercial duck. Poult Sci. Aug; 100(8): 101248. doi: 10.1016/j.psj.2021.101248. Epub 2021 May 13. PMID: 34225201; PMCID: PMC8264152.

21. Shidham VB, Swami VK. 2000. Evaluation of apoptotic leukocytes in peripheral blood smears. Arch. Pathol. Lab. Med. 124: 1291-1294. doi: 10.1043/0003-9985(2000)124<1291: EOALIP>2.0.CO;2.

22. Scanes CG. 2016. Biology of stress in poultry with emphasis on glucocorticoids and the heterophil to lymphocyte ratio. Poult. Sci. 95: 2208-2215 http://dx.doi.org/10.3382/ps/pew137

23. Davis AK, Maney DL, Maerz JC. 2008. The use of leukocyte profiles to measure stress in vertebrates: a review for ecologists. Functional Ecology 22:760-772 https://doi.org/10.1111/j.13652435.2008.01467.x. 